\title{
ACUTE LARVICIDAL TOXICITY AND REPELLENCY EFFECT OF OCTOPUS CYANEA CRUDE EXTRACTS AGAINST THE FILARIASIS VECTOR, CULEX PIPIENS
}

\author{
By \\ HUSSEIN A. EL-NAGGAR and AHMED I. HASABALLAH ${ }^{\star}$ \\ Department of Zoology, Faculty of Science, Al-Azhar University, Cairo, Egypt \\ ( ${ }^{\star}$ Correspondence: ahscience09@azhar.edu.eg)
}

\begin{abstract}
This study evaluated the acute larval toxicity and repellent effect of solvent extracts of the big blue octopus, Octopus cyanea on the mosquito, Culex pipiens. Results showed that the highest effect of the acute larvicidal activities was recorded for ethanol extract $\left(\mathrm{LC}_{50}=24.57 \mathrm{ppm}\right)$, followed by methanol extract $\left(\mathrm{LC}_{50}=35.85 \mathrm{ppm}\right)$. The repellency percentages increased with the increase of concentration producing the highest at $200 \mathrm{ppm}$ and $50 \mathrm{ppm}$ for methanol and ethanol extracts, respectively. GC-MS analysis revealed the presence of 19 compounds in ethanol extract and 5 compounds in methanol extract with insecticidal activities. The major identified bioactive compounds are Eugenol; Furoscrobiculin B; Hexadecanoic acid, methylester; Hexadecanoic acid, 1(hydroxymethyl)-1,2-ethanediylester; Octadecanoic acid, methylester; Oleic acid, eicosyl ester; 2-Methyl-5H-dibenz [b, f] azepine and many polysiloxanes compounds. Generally, results obtained indicated that $O$. cyanea ethanol extract induced remarkable effects on both acute larvicidal and repellent activities.
\end{abstract}

Keywords: Culex pipiens; toxicity; repellency; octopus; GC-MS.

\section{Introduction}

Mosquitoes play a considerable role as vectors of a wide variety of human pathogens and parasites. Culex pipiens is one of the prevalent mosquitoes in Egypt. It is the vector of filariasis (Fouda et al, 2013), Rift Valley fever virus and West Nile virus (El-Bahnasawy et al, 2013a,b) and other diseases like leishmaniasis, dengue and malaria that cause a considerable morbidity, mortality and economic load; (El-Kholy et al, 2017) reported possible role in the transmission of $\mathrm{HC}$ virus. Control of mosquitoes largely relies on chemical insecticides. Unfortunately, mosquitoes have developed resistance to most of synthetic insecticides (Chareonviriyaphap et al, 2013); thus the control of mosquito vector encounters a number of major difficulties, besides the adverse effects of chemical insecticides on the environment and health (Kumar et al, 2012). In the same context, searching for new compounds from natural origin for their mosquitocidal activities may replace conventional insecticides and these compounds may serve as insecticides, antifeedants as well as repellents (Reegan et al, 2013; Hasaballah and El-Naggar, 2017).
Marine organisms considered a source for Marine organisms considered a source for numerous bioactive compounds and secondary metabolites that attract the attention of chemists and biologists due to their potential anticancer, antimicrobial, antiviral and antifungal activities (Zapata and Amemiya, 2000; Datta et al, 2015). Environmental challenges such as nutrition, space competition, predation and self-defence stimulate marine organisms to produce secondary metabolites. Marine invertebrates have the ability to live in halobiotic environment; special adaptation, metabolic activities and secretions propel them to produce bioactive substances to combat these environmental challenges. The whole body extract of several marine organisms showed a potential therapeutic source for various diseases (Datta et al, 2015).

An alternative insect and/or pest control method is the utilization of marine natural products to reduce the toxic insecticide usage and minimize harmful impact on insects and also on the environment (Dias et al, 2012; Hasaballah and El-Naggar, 2017). As a result, many scientists focused on isolation of compounds with insecticidal properties from marine algae (El-Sayed et al, 1997), sponge 
(Hasaballah and El-Naggar, 2017; Elnagar et al, 2018), soft corals (Momtaz, 2016) and marine microorganisms (Xiong et al, 2004).

Marine organisms, including Molluscs proved their potential role as a source for various natural products with multiple bioactivities (Gnanambal et al, 2005; Li et al, 2009; Benkendorff 2010). Natural products research on the phylum Mollusca were primarily focused on the shell-less or soft-bodied molluscs, particularly cephalopoda, nudibranchs and opisthobrachs (Karuso, 1987; Faulkner, 1992; Babar et al, 2012; Ióca et al, 2018; Miller et al, 2018). Cephalopods such as cuttlefishes, squids, nautilus and octopus are the main important sources of such bioactive compounds. As the majority of them lack an outer shell, they use a variety of defense ways, including venom to neutralize enemies and prey, and liquid-ink to evade predator attacks (Sudhakar and Nazeer, 2015).

This study was made as an attempt to investigate the acute larvicidal activity and repellent effect of ethanolic and methanolic extracts of the big blue octopus, Octopus cyanea against mosquito vector, Culex pipiens.

\section{Materials and Methods}

Mosquito, C. pipiens Linnaeus (L) was obtained from Medical Entomology Research Center. It was reared for several generations, in the insectary of medical entomology at the department of zoology, faculty of science, AlAzhar University, under controlled conditions at a temperature of $27 \pm 2^{\circ} \mathrm{C}$, with relative humidity of $70 \pm 10 \%$ and 12-12 hr light-dark regimen. Adult mosquitoes were kept in wooden cages and daily provided with sponge pieces soaked in $10 \%$ sucrose solution for a period of 3-4 days after emergence. After this period the females were allowed to take a blood meal from a pigeon host, which is necessary for laying eggs (Hasaballah 2015).

The octopus specimens were collected by hand from reef flat crevices during spring 2017 from Napq protected area at Aqaba Gulf coast of Egypt. After collection, the specimens were washed and preserved at $-20^{\circ} \mathrm{C}$ in an icebox filled with ice cubes and some of table salt for late processing in the laboratory.
By using keys proposed in Vine (1986), Erwin and Picton (1990), and Lieske and Myers (2004), the specimens' identification was done on the basis of their morphological features.

The big blue octopus $O$. cyanea, belong to Phylum: Mollusca, Class: Cephalopoda, Order: Octopoda, Family: Octopodidae. It is commonly found in coral reefs and shallow water at depths of 1 to 100 meters in Pacific Ocean, Red Sea and Mediterranean Sea. It is a diurnally active predator searches for fish, crabs, shrimp and molluscs (Mather and Mather, 2004).

Preparation of crude extract: In the laboratory, the samples were washed under running tap water and chopped into small pits. To prepare the crude methanol and ethanol extracts, two samples of about $200 \mathrm{~g}$ were set in two bottles, the first was homogenized with $400 \mathrm{ml} \mathrm{70 \%} \mathrm{aqueous} \mathrm{ethanol} \mathrm{and} \mathrm{the} \mathrm{second}$ was homogenized with $400 \mathrm{ml}$ absolute methanol. The mixtures were continuously stirred and stored in dark to avoid photolysis and secondary metabolites degradation at room temperature. After a week with gentle shaking, mixtures were filtered via Whatman 542 filter paper. Solvents were evaporated with rotary evaporator (Ballantine et al, 1987).

Different concentrations were prepared to study the acute larvicidal and repellent activities of tested extracts. The early 3rd larval instar was collected and placed in plastic cup its capacity $250 \mathrm{~mL}$ containing the extract solution. Control larvae were placed in $250 \mathrm{~mL}$ distilled water ( 25 of 3 rd instar larvae/cup).

Acute larvicidal toxicity: The acute larvicidal toxicity that measured as mortality recorded $24 \mathrm{~h}$ post treatment was determined for the early $3^{\text {rd }}$ larval instar of $C x$. pipiens. Mosquito larvicidal toxicity assays were done (WHO, 1996) with some modifications (Pavela et al, 2016). Dilution of extracts was carried out in dimethyl sulphoxide (DMSO) to prepare a serial dilution of test concentrations. Early $3^{\text {rd }}$ larval instar was selected and transferred in $25 \mathrm{~mL}$-distilled water. For each treatment, $1 \mathrm{~mL}$ of serial dilutions was added to $224 \mathrm{~mL}$ of distilled water and lightly shak- 
en to ensure a homogenous test solution. The selected larvae were transferred in distilled water into a cup of prepared test solution (25larvae/cup). Three replicates were used in each experiment with at least 6 concentrations. During the experiments, food was not offered to the mosquito larvae.

Repellent activities: Selected concentrations from each extract were dissolved in $2 \mathrm{~mL}$ water with a drop of Tween 8 . The concentration was directly applied onto ventral surface of pigeon after abdominal feathers removal. Positive control tests were carried out using commercial repellent (Deet) purchased from Johnson Wax, Egypt, after (Uniyal et al, 2016). For each experimental treatment and after $10 \mathrm{~min}$, pigeon was placed for $2 \mathrm{hr}$ in cage containing fifty starved $C$. pipiens adult females. Alongside with these treatments control test was carried out using water. The number of fed and unfed females were counted and calculated after treatments.

GC-MS analysis: Gas Chromatography Mass Spectrometry (GC-MS) analysis was performed using gas Hewlett-Packard HP5890 series II equipped with split/splitless injector and a capillary column $(30 \mathrm{~m}, 0.25$ $\mathrm{mm}$ and $0.25 \mathrm{~lm}$ ) fused with phenyl poly (silphenylenesiloxane). The temperature of both injector and detector was set at 280 and 300 ${ }^{\circ} \mathrm{C}$, respectively; the oven temperature was kept at $80^{\circ} \mathrm{C}$ for $1 \mathrm{~min}$ up to $300^{\circ} \mathrm{C}$ at $20^{\circ} \mathrm{C} /$ min. Helium was used as carrier gas at continuous and constant flow of $1.0 \mathrm{ml} / \mathrm{min}$. A volume of $2 \mu \mathrm{l}$ was injected into the splitless mode and $1 \mathrm{~min}$ purge. The MS (HewlettPackard 5889B MS Engine) was used with selected ion monitoring (SIM). The mass spectrometer was operated at $70 \mathrm{eV}$ and scan fragments from 50 to $650 \mathrm{~m} / \mathrm{z}$. The crude extract peak identification was performed depending on the obtained mass spectra with those available in the NIST library.

Statistical analysis: Abbott's formula (Abbott, 1925) was used to correct control mortality (which was <3\%). The larval mortality data were subjected to probit analysis for calculating $\mathrm{LC}_{50}, \mathrm{LC}_{90}, 95 \%$ confidence limits and Chi-square values were calculated using sta- tistical package for social sciences (SPSS, ver. 15.0). The repellency percent was calculated by the following formula, Repellency $\%$ $=[\mathrm{A} \%-\mathrm{B} \% / 100-\mathrm{B} \%] \times 100$, where $\mathrm{A}$ was percent of unfed females in treatment and $B$ percent of unfed females in control.

\section{Results}

The acute larvicidal toxicities of $O$. cyanea different extracts on the early $3^{\text {rd }}$ instar $C$. pipiens larvae were tested and the $\mathrm{LC}_{50}, \mathrm{LC}_{90}$, confidence limits at $(95 \%)$, regression equation and chi-square were also calculated (Tables 1,2).

The acute larvicidal toxicity of the $3^{\text {rd }}$ larval instar of $C x$. pipiens treated with $O$. cyanea crude methanol extract increased gradually as the applied concentration increased when compared to the control group. The highest acute toxicity \% recorded was 98.7 and 90.7 at concentrations 200 and $100 \mathrm{ppm}$, respectively compared to $2.7 \%$ for control (Tab. 1).

Data revealed that the acute larvicidal toxicity of the $3^{\text {rd }}$ larval instar of $C$. pipiens treated with the crude ethanol extract of $O$. cyanea was concentration-dependent (i.e. increased as the concentration increased). The highest acute toxicity \% recorded was 98.7 and 86.7 at concentrations 50 and $40 \mathrm{ppm}$, respectively compared to $0.0 \%$ for the control group (Tab. 2).

Testing the effect of $O$. cyanea different crude extracts against the acute larvicidal toxicity of the $3^{\text {rd }}$ instar $C$. pipiens larvae showed that the highest effect was recorded by $O$. $c y$ anea ethanol extract. Based on the comparison of the lethal concentrations, ethanol extract was selected as the most effective, $\mathrm{LC}_{50}$ recorded $(24.57 \mathrm{ppm})$, followed by me-thanol extract $(35.85 \mathrm{ppm})$ and $\mathrm{LC}_{90}$ recorded $(43.89$ ppm), followed by methanol extract (145.23 ppm).

Repellent effects of $O$. cyanea crude methanol extract against starved $C$. pipiens adult females. The repellent effect was concentration-dependent (i.e. increased as the concentration increased). At concentrations $100 \&$ $200 \mathrm{ppm}$, repellent effect was extremely induced as calculated in $84 \& 87.94 \%$, respec- 
tively, compared to $100 \%$ repellent for DEET at a concentration 10 ppm (Tab. 3).

Repellent effect of different concentrations of crude ethanol extract against starved $C x$. pipiens females showed that effect was concentration-dependent, at $>40 \mathrm{ppm}$, was extremely induced. Compared with ethanol extract was higher against $C x$. pipiens followed by methanol extract that effect depended on extract concentration strength (Tab. 4).

The GC-MS analysis of $O$. cyanea ethanol extract recorded nineteen active compounds with insecticidal activities. The most predominant compounds are Furoscrobiculin B; Eugenol; Phenol, 2-methoxy-3- (2-propenyl); Hexadecanoic acid, methylester; Hexadecanoic acid, 1-(hydroxymethyl)-1,2-ethanediyl ester; Octadecanoic acid, methylester, Cyclodecasiloxane, eicosamethyl; Oleic acid, eicosyl ester; trans-Isoeugenol; Phenol, 2-methoxy-5-(1-propenyl)-(E); Docosanoic acid,1,2, 3-propanetriyl ester; Octasiloxane, 1,1,3,3,5,
5,7,7,9,9,11, 11,13,13,15,15-hexadecamethyl; 2-Methyl-5H-dibenz[b,f]azepine; Cyclotetrasiloxane, octamethyl; Pyrazole[4,5-b]imidazole,1-formyl-3-ethyl-6-á-d-ribofuranosyl;

Cyclohexasiloxane, dodecamethyl; Cycloheptasiloxane, tetradecamethyl; Hexasiloxane, 1,1,3-,3,5,5,7,7,9,9,11,11-dodecamethyl; Cyclo-nonasiloxane and octadecamethyl with peak areas of $11.78 ; 11.09 ; 11.09 ; 5.37 ; 5.17$; $4.76 ; 4.29 ; 3.17 ; 1.86 ; 1.86 ; 1.17 ; 1.23 ; 0.7$; $0.42 ; 0.19,0.06,0.6,0.6$ and $0.22 \%$, respectively (Tab. 5).

The GC-MS analysis of $O$. cyanea methanol extract revealed five active compounds with insecticidal activities. These compounds are, Cyclodecasiloxane, eicosamethyl; Cycloheptasiloxane, tetradecamethyl; Octasiloxane, $1,1,3,3,5,5,7,7,9,9,11,11,13,13,15,15-$ hexadecamethyl; Cyclooctasiloxane, hexadecamethyl; Cyclohexasiloxane, dodecamethyl, with low peak areas of $1.50 ; 0.67 ; 0.67$; 0.35 and $0.25 \%$, respectively (Tab. 6).

\begin{tabular}{|c|c|c|c|c|c|}
\hline $\begin{array}{c}\text { Concentrations } \\
\text { ppm }\end{array}$ & $\begin{array}{c}\text { Acute larvicidal } \\
\text { toxicity } \%\end{array}$ & $\begin{array}{c}\mathrm{LC}_{50} \text { ppm } \\
\text { (Its limits at 95\%) }\end{array}$ & $\begin{array}{c}\mathrm{LC}_{90} \text { ppm } \\
\text { (Its limits at } 95 \% \text { ) }\end{array}$ & $\begin{array}{c}\text { Regression } \\
\text { equation }\end{array}$ & $\chi^{2}$ \\
\hline 200 & 98.7 & \multirow{7}{*}{$\begin{array}{c}35.85 \\
(34.54-38.21)\end{array}$} & \multirow{7}{*}{$\begin{array}{c}145.23(143.53- \\
150.42)\end{array}$} & \multirow{7}{*}{$\begin{array}{c}\mathrm{Y}=0.3657 \mathrm{x}+ \\
36.889\end{array}$} & \multirow{7}{*}{2.241} \\
\hline 100 & 90.7 & & & & \\
\hline 50 & 65.3 & & & & \\
\hline 25 & 54.7 & & & & \\
\hline 12.5 & 38.7 & & & & \\
\hline 6.25 & 17.3 & & & & \\
\hline Control & 2.7 & & & & \\
\hline
\end{tabular}

Table 2: Larvicidal toxicity of $O$. cyanea crude ethanol extract against $3^{\text {rd }}$ larval instar of Cx. pipiens

\begin{tabular}{|c|c|c|c|c|c|}
\hline $\begin{array}{c}\text { Concentrations } \\
\text { ppm }\end{array}$ & $\begin{array}{c}\text { Acute larvicidal } \\
\text { toxicity } \%\end{array}$ & $\begin{array}{c}\mathrm{LC}_{50} \mathrm{ppm} \\
\text { (Its limits at } 95 \% \text { ) }\end{array}$ & $\begin{array}{c}\mathrm{LC}_{90} \mathrm{ppm} \\
\text { (Its limits at 95\%) }\end{array}$ & $\begin{array}{l}\text { Regression } \\
\text { equation }\end{array}$ & $\chi^{2}$ \\
\hline 50 & 98.7 & \multirow{8}{*}{$\begin{array}{c}24.57 \\
(22.40-28.08)\end{array}$} & \multirow{8}{*}{$\begin{array}{c}43.89 \\
(41.71-47.96)\end{array}$} & \multirow{8}{*}{$\begin{array}{l}Y=2.0696 \\
x-0.8518\end{array}$} & \multirow{8}{*}{1.193} \\
\hline 40 & 86.7 & & & & \\
\hline 30 & 69.3 & & & & \\
\hline 20 & 30.7 & & & & \\
\hline 10 & 14.7 & & & & \\
\hline 5 & 9.3 & & & & \\
\hline 2.5 & 10.7 & & & & \\
\hline Control & 0.0 & & & & \\
\hline
\end{tabular}

Table 3: Repellency effect of $O$. cyanea crude methanol extract against $C x$. pipiens females

\begin{tabular}{|c|c|c|c|c|c|c|}
\hline Concentrations ppm & Females & Fed No. & Fed\% & Unfed No. & Unfed \% & Repellency \% \\
\hline 200 & 50 & 3.0 & 6.0 & 47 & 94 & 87.94 \\
\hline 100 & 50 & 8.0 & 16 & 42 & 84 & 84 \\
\hline 50 & 50 & 21 & 42 & 29 & 58 & 58 \\
\hline 25 & 50 & 30 & 60 & 20 & 40 & 40 \\
\hline 12.5 & 50 & 42 & 84 & 8 & 16 & 16 \\
\hline 6.25 & 50 & 45 & 90 & 5.0 & 10 & 10 \\
\hline Deet (10 ppm) & 50 & 0.0 & 0.0 & 50 & 100 & 100 \\
\hline Control & 50 & 47 & 94 & 3.0 & 6.0 & 6.0 \\
\hline
\end{tabular}


Table 4: Repellency effect of $O$. cyanea crude ethanol extract against $C x$. pipiens females

\begin{tabular}{|c|c|c|c|c|c|c|}
\hline Concentrations ppm & Females & Fed No. & Fed $\%$ & Unfed No. & Unfed \% & Repellency $\%$ \\
\hline 50 & 50 & 1.0 & 2.0 & 49 & 98 & 93.96 \\
\hline 40 & 50 & 3.0 & 6.0 & 47 & 94 & 94 \\
\hline 30 & 50 & 9.0 & 18 & 41 & 82 & 82 \\
\hline 20 & 50 & 19 & 38 & 31 & 62 & 62 \\
\hline 10 & 50 & 32 & 64 & 18 & 36 & 36 \\
\hline 5.0 & 50 & 41 & 82 & 9.0 & 18 & 18 \\
\hline 2.5 & 50 & 48 & 96 & 2.0 & 4.0 & 4.0 \\
\hline Deet (10 ppm) & 50 & 0.0 & 0.0 & 50 & 100 & 100 \\
\hline Control & 50 & 48 & 96 & 2.0 & 4.0 & 4.0 \\
\hline
\end{tabular}

Table 5: Compounds with insecticidal activities from GC-MS analysis of $O$. cyanea ethanol extract

\begin{tabular}{|c|c|c|c|c|c|c|}
\hline Compound Name & RT & Area \% & Formula & $\begin{array}{c}\text { Mo. } \\
\text { Weight }\end{array}$ & $\begin{array}{l}\text { Compound } \\
\text { Nature }\end{array}$ & Activity \\
\hline Furoscrobiculin B & 38.17 & 11.78 & $\mathrm{C} 15 \mathrm{H} 20 \mathrm{O} 2$ & 232 & $\begin{array}{c}\text { Lactarane } \\
\text { sesquiterpenes }\end{array}$ & Antifeedant \\
\hline Eugenol & 24.93 & 11.09 & $\mathrm{C} 10 \mathrm{H} 12 \mathrm{O} 2$ & 164 & $\begin{array}{l}\text { Phenolic } \\
\text { compound }\end{array}$ & $\begin{array}{l}\text { Insecticide, pesticide, } \\
\text { larvicide, repellent }\end{array}$ \\
\hline Phenol, 2-methoxy-3-(2-propenyl) & 24.93 & 11.09 & $\mathrm{C} 10 \mathrm{H} 12 \mathrm{O} 2$ & 164 & $\begin{array}{l}\text { Phenolic } \\
\text { compound }\end{array}$ & $\begin{array}{l}\text { Insecticide pesticide, } \\
\text { larvicide, repellent }\end{array}$ \\
\hline Hexadecanoic acid, methylester & 38.01 & 5.37 & $\mathrm{C} 17 \mathrm{H} 34 \mathrm{O} 2$ & 270 & $\begin{array}{l}\text { Palmitic } \\
\text { acid ester }\end{array}$ & Pesticide \\
\hline $\begin{array}{l}\text { Hexadecanoic acid, 1-(hydroxymethyl)-1,2- } \\
\text { ethanediyl ester }\end{array}$ & 39.53 & 5.17 & $\mathrm{C} 35 \mathrm{H} 68 \mathrm{O} 5$ & 568 & Fatty acid esters & Pesticide \\
\hline Octadecanoic acid, methylester & 42.03 & 4.76 & $\mathrm{C} 19 \mathrm{H} 38 \mathrm{O} 2$ & 298 & Fatty acid esters & Repellent \\
\hline Cyclodecasiloxane, eicosamethyl & 47.55 & 4.29 & C20H60O10Si10 & 740 & \begin{tabular}{|c|}
$\begin{array}{c}\text { Silicone } \\
\text { polymers }\end{array}$ \\
\end{tabular} & $\begin{array}{l}\text { Surfactant in certain } \\
\text { pesticide products }\end{array}$ \\
\hline Oleic acid, eicosyl ester & 41.55 & 3.17 & $\mathrm{C} 38 \mathrm{H} 74 \mathrm{O} 2$ & 562 & $\begin{array}{c}\text { Ester derivatives } \\
\text { of fatty acids }\end{array}$ & $\begin{array}{l}\text { Insecticide, repellent, } \\
\text { larvicide }\end{array}$ \\
\hline Trans-Isoeugenol & 26.54 & 1.86 & $\mathrm{C} 10 \mathrm{H} 12 \mathrm{O} 2$ & 164 & $\begin{array}{c}\text { Phenolic } \\
\text { compound }\end{array}$ & $\begin{array}{l}\text { Insecticide, pesticide, } \\
\text { larvicide, repellent }\end{array}$ \\
\hline Phenol, 2-methoxy-5-(1-propenyl)-(E) & 26.54 & 1.86 & $\mathrm{C} 10 \mathrm{H} 12 \mathrm{O} 2$ & 164 & $\begin{array}{l}\text { Phenolic } \\
\text { compound }\end{array}$ & $\begin{array}{l}\text { Insecticide, pesticide, } \\
\text { larvicide, repellent }\end{array}$ \\
\hline $\begin{array}{c}\text { Octasiloxane, } 1,1,3,3,5,5,7,7,9,9,11,11,13,13,15,15 \\
\text { hexadecamethyl }\end{array}$ & 40.36 & 1.17 & $\mathrm{C} 16 \mathrm{H} 50 \mathrm{O} 7 \mathrm{Si} 8$ & 578 & \begin{tabular}{|c|}
$\begin{array}{c}\text { Silicone } \\
\text { polymers }\end{array}$ \\
\end{tabular} & $\begin{array}{l}\text { Surfactant in certain } \\
\text { pesticide products }\end{array}$ \\
\hline Docosanoic acid,1,2,3- propanetriyl ester & 41.68 & 1.23 & C69H134O6 & 1058 & Fatty acid esters & Pesticide \\
\hline 2-Methyl-5H-dibenz[b,f]azepine & 9.62 & 0.7 & $\mathrm{C} 15 \mathrm{H} 13 \mathrm{~N}$ & 207 & \begin{tabular}{|c|c|}
$\begin{array}{c}\text { Azepine } \\
\text { derivatives }\end{array}$ \\
\end{tabular} & Insecticide \\
\hline Cyclotetrasiloxane, octamethyl & 25.68 & 0.42 & $\mathrm{C} 10 \mathrm{H} 12 \mathrm{O} 2$ & 164 & $\begin{array}{l}\text { Silicone } \\
\text { polymers }\end{array}$ & $\begin{array}{l}\text { Surfactants in certain } \\
\text { pesticide products }\end{array}$ \\
\hline $\begin{array}{l}\text { Pyrazole[4,5-b]imidazole,1-formyl-3-ethyl-6-á-d- } \\
\text { ribofuranosyl }\end{array}$ & 18.07 & 0.19 & $\mathrm{C} 12 \mathrm{H} 16 \mathrm{~N} 4 \mathrm{O} 5$ & 296 & $\begin{array}{l}\text { Pyrazole } \\
\text { Derivatives }\end{array}$ & $\begin{array}{l}\text { Insecticide, pesticide, } \\
\text { larvicide }\end{array}$ \\
\hline Cyclohexasiloxane, dodecamethyl & 22.17 & 0.06 & $\mathrm{C} 12 \mathrm{H} 36 \mathrm{O} 6 \mathrm{Si} 6$ & 444 & $\begin{array}{l}\text { Silicone } \\
\text { polymers }\end{array}$ & $\begin{array}{c}\text { Surfactant in certain } \\
\text { pesticide products }\end{array}$ \\
\hline Cycloheptasiloxane, tetradecamethyl & 26.74 & 0.6 & $\mathrm{C} 14 \mathrm{H} 42 \mathrm{O} 7 \mathrm{Si} 7$ & 518 & $\begin{array}{l}\text { Silicone } \\
\text { polymers }\end{array}$ & $\begin{array}{c}\text { Mosquitocide, surfactant } \\
\text { in certain pesticide } \\
\text { products }\end{array}$ \\
\hline $\begin{array}{c}\text { Hexasiloxane, } 1,1,3,3,5,5,7,7,9,9,11,11- \\
\text { dodecamethyl }\end{array}$ & 26.74 & 0.6 & $\mathrm{C} 12 \mathrm{H} 38 \mathrm{O} 5 \mathrm{Si} 6$ & 430 & $\begin{array}{l}\text { Silicone } \\
\text { polymers }\end{array}$ & $\begin{array}{l}\text { Surfactant in certain } \\
\text { pesticide products }\end{array}$ \\
\hline $\begin{array}{c}\text { Cyclononasiloxane, } \\
\text { octadecamethyl }\end{array}$ & 34.37 & 0.22 & C18H54O9Si9 & 666 & $\begin{array}{l}\text { Silicone } \\
\text { polymers }\end{array}$ & $\begin{array}{l}\text { Surfactant in certain } \\
\text { pesticide products }\end{array}$ \\
\hline
\end{tabular}

Table 6: Compounds with insecticidal activities from GC-MS analysis of $O$. cyanea methanol extract

\begin{tabular}{|c|c|c|c|c|c|c|}
\hline Compound Name & RT & Area $\%$ & Formula & $\begin{array}{c}\text { Mo. } \\
\text { Weight }\end{array}$ & $\begin{array}{l}\text { Compound } \\
\text { Nature }\end{array}$ & Activity \\
\hline $\begin{array}{l}\text { Cyclodecasiloxane, } \\
\text { eicosamethyl }\end{array}$ & 49.38 & 1.50 & C20H60O10Sil0 & 740 & $\begin{array}{l}\text { Silicone } \\
\text { polymers }\end{array}$ & $\begin{array}{l}\text { Surfactant in certain } \\
\text { pesticide products }\end{array}$ \\
\hline $\begin{array}{l}\text { Cycloheptasiloxane, } \\
\text { tetradecamethyl }\end{array}$ & 26.71 & 0.67 & $\mathrm{C} 14 \mathrm{H} 42 \mathrm{O} 7 \mathrm{Si} 7$ & 518 & $\begin{array}{l}\text { Silicone } \\
\text { polymers }\end{array}$ & $\begin{array}{l}\text { Mosquitocide, surfactants } \\
\text { in certain pesticides }\end{array}$ \\
\hline $\begin{array}{l}\text { Octasiloxane, } 1,1,3,3,5,5,7,7,9,9,1 \\
1,11,13,13,15,15 \text {-hexadecamethyl }\end{array}$ & 26.71 & 0.67 & $\mathrm{C} 16 \mathrm{H} 50 \mathrm{O} 7 \mathrm{Si} 8$ & 578 & $\begin{array}{l}\text { Silicone } \\
\text { polymers }\end{array}$ & $\begin{array}{l}\text { Surfactant in certain } \\
\text { pesticides }\end{array}$ \\
\hline $\begin{array}{l}\text { Cyclooctasiloxane, } \\
\text { hexadecamethyl }\end{array}$ & 30.82 & 0.35 & C16H48O8Si8 & 592 & $\begin{array}{l}\text { Silicone } \\
\text { polymers }\end{array}$ & $\begin{array}{l}\text { Surfactant in certain } \\
\text { pesticides }\end{array}$ \\
\hline Cyclohexasiloxane, dodecamethyl & 22.11 & 0.25 & $\mathrm{C} 12 \mathrm{H} 36 \mathrm{O} 6 \mathrm{Si} 6$ & 444 & $\begin{array}{l}\text { Silicone } \\
\text { polymers }\end{array}$ & $\begin{array}{l}\text { Surfactant in certain } \\
\text { pesticides }\end{array}$ \\
\hline
\end{tabular}

\section{Discussion}

Mosquito control at the larval stage proved an effective tool for mosquito-borne diseases (Nandita et al, 2008). Also, environmental safety is the most important issue in mosquito control programs (Wafa et al, 2014). The use of natural products in mosquito control to prevent the proliferation of mosquito-borne diseases and to protect the environment from the application of chemical insecticides and pesticides is the main goal for scientists.

In the present results, GC-MS analysis revealed 19 active compounds in ethanol extract, while methanol extract possesses 5 active compounds. GC-MS results confirmed the higher efficacy of ethanol extract against 
mosquito vector. The identified compounds in ethanol extract recorded activity against insects; some as pesticides; hexadecanoic acid, methylester (Jemimma et al, 2017), hexadecanoic acid, 1-(hydroxymethyl)-1,2-ethanediyl ester (Niyogi et al, 2016) and docosanoic acid, 1,2,3-propanetriyl ester (Geetha et al, 2013), Others act as insecticide; 2-Methyl-5 H-dibenz [b, f] azepine (Panneerselvam et al, 2012), or as repellents; octadecanoic acid, methylester (Abubakar and Majinda, 2016) and others have many activities; eugenol; transisoeugenol; phenol, 2-methoxy-5-(1-propen-yl)- and phenol, 2-methoxy-3-(2-propenyl) (Keg-ley et al, 2010), and others have insecticide, pesticide, larvicide and repellent properties; oleic acid, eicosyl ester (Gurunathan $e t$ $a l, 2016)$. Ethanol extract was rich with compounds act as surfactants in certain pesticides; cyclodecasiloxane, eicosamethyl; cyclotetrasiloxane, octamethyl; cyclohexasiloxane, dodecamethyl; cycloheptasiloxane, tetradecamethyl; cyclononasiloxane, octadecamethyl; octasiloxane, 1,1,3,3,5,5,7,7,9,9,11,11,13,13, 15,15 hexadecamethyl \& hexasiloxane, 1,1,3, $3,5,5,7,7,9,9,11,11$ dodecamethyl. But, methanol extract produces compounds act as surfactants in certain pesticide products and only cycloheptasiloxane and tetradecamethyl with insecticidal activities (Jayashree et al, 2015).

One of the commonest compounds with insecticidal, pesticidal, larvicidal and repellent activities is eugenol (phenylpropene) is a potential alternative to synthetic insecticides due to lower mammalian toxicity, rapid degradation in environment and complex mixture of bioactive constituents with multi-modal activities against target insects (Kegley et al, 2010). Phenolic compounds widely scattered in ethanol extract have substantial allelopathic applications in agriculture and forestry as herbicides, insecticides and fungicides (Zhao et al, 2010; Bhawan and Nagar, 2016). The second major compound is Furoscrobiculin$\mathrm{B}$, a lactarane sesquiterpenes compound possesses antifeedant activity (Ogino et al, 1994; Catelan et al, 2015).

The biological activity of oleic acid, eicosyl ester uncovered the reasonable larvicidal and repellent activities due to the presence of certain chitinase inhibitors and ecdysone-20monooxygenase. This effect may be attributed to the dechitinization of body wall and inhibition of ecdysone-20-monooxygenase, an enzyme required to promote cell membrane development. Gurunathan et al. (2016) confirmed that these compounds could be considered as a potent source for their mosquito larvicidal properties. Among the identified photo-chemicals, Hexadecanoic acid; methylester; Hexadecanoic acid; 1-(hydroxymethyl)-1,2-ethanediyl ester; Octadecanoic acid; methylester and docosanoic acid, 1,2,3-propanetriyl ester have antioxidant, nematocide and pesticide activities (Gopalakrishnan and Vadivel, 2011; Geetha et al, 2013).

Pyrazole derivatives are intermediate in biological materials. They have a history of application in agrochemical industry as herbicides, insecticides \& acaricides. GC-MS analysis showed that ethanol extract contains pyrazole [4,5-b] imidazole, 1-formyl-3-ethyl-6-ád-ribofuranosyl. Song et al. (2013) showed that pyrazole derivatives possess promise activities against many insects as bean aphid Aphis craccivora, CX. pipiens and moth Plutella xylostella. Dibenzoazepine derivative proved effective against several diseases and pathogens. The 2-Methyl-5H-dibenz [b, f] azepine only derivative was found in ethanol extract. Azepine derivatives have some activities as antiviral, anticancer \& insecticidal activity (Panneerselvam et al, 2012).

Inhibitory effect of ethanol extract against $C x$. pipiens was related to excessive amount of polysiloxanes. Silicone polymers are widely used as surfactants in certain pesticides. Many polysiloxanes were found in tested extracts, but in ethanol extract in massive amounts. Polysiloxanes are important bioactive compounds with insecticidal properties (Singh and Handique, 1997).

\section{Conclusion}

The highest acute larvicidal activity was the ethanol extract followed by methanol extract. The repellency percentages increased as the applied concentration increased. These results showed that extracts of new naturally comp- 
ounds and their derivatives proved to be effective, cheap and environmental friend insecticides against mosquito-borne diseases.

\section{References}

Abbott, WS, 1925: A method for computing the effectiveness of an insecticide. J. Eco. Entomol. 18:265-77.

Abubakar, MN, Majinda, RRT, 2016: GC-MS analysis and preliminary antimicrobial activity of Albizia adianthifolia (Schumach) and Pterocarpus angolensis (DC). Medicines 3, 1: 3.

Babar, AG, Pande, A, Kulkarni, BG, 2012: Bioactive potential of some intertidal molluscs collected from Mumbai coast, West coast of India. Asian Pac. J. Trop. Biomed. 2, 2:S1060-3.

Ballantine, DL, Gerwick, WH, Velez, SM, Alexander, E, Guevara, P, 1987: Antibiotic activity of lipid-soluble extracts from Caribbean marine algae. Hydrobiol. 151/152:463-9.

Benkendorff, K, 2010: Molluscan biological and chemical diversity: secondary metabolites and medicinal resources produced by marine molluscs. Biol. Rev. 85:757-75.

Bhawan, P, Nagar, EA, 2016: Phenols \& Phenolic compounds. Central pollution control board (Ministry of Environment, Forests \& Climate Change). Delhi-110032: 72.

Catelan, TBS, de Arruda, EJ, Oliveira, LCS, Raminelli, C, Gaban, CRG, et al, 2015: Evaluation of toxicity of phenolic compounds using Aedes aegypti (Diptera: Culicidae) and Artemia salina. Adv. Infect. Dis. 5:48-56.

Chareonviriyaphap, T, Bangs, MJ, Suwonkerd, W, Kongmee, M, Corbel, V, et al, 2013: Review of insecticide resistance and behavioral avoidance of vectors of human diseases in Thailand. Parasit. Vectors 6:280-6.

Datta, D, Talapatra, NS, Swarnakar, S, 2015: Bioactive compounds from marine invertebrates for potential medicines: An overview. Int. Lett. Nat. Sci. 34:42-61.

Dias, DA, Urban, S, Roessner, U, 2012: A Historical Overview of Natural Products in Drug Discovery. Metabolites 2: 303-36.

El-Bahnasawy, MM, Khater, MK, Morsy, TA, 2013b: The mosquito borne west Nile virus infection: Is it threating to Egypt or a neglected endemic disease? J. Egyptian. Soc. Parasitol. 43, 1:87-102.

El-Bahnasawy, MM, Megahed, LA, Saleh, HA, Morsy, TA, 2013a: The Rift Valley fever: Could re-emerge in Egypt again? J. Egyptian. Soc. Parasitol. 43, 1:41-56.
El-Kholy, SE, El-Husseiny, IM, Meshrif, WS, El-Azm, AA, Salem, ML, 2017: Does the mosquito Culex pipiens represent a potential vector of hepatitis C virus? Med. Vet. Entomol. 32:155-61. El-Sayed, KA, Dunbar, DC, Perry, TL, Wilkins, SP, Hamann, MT, et al, 1997: Marine natural products as prototype insecticidal agents. J. Agric. Food Chem. 45:2735-9.

Elnagar, HM, Soliman, MHA, El-Naggar, HA, Bashar, MAE, 2018: The Toxic Effect of Certain New Alternative Insecticides against Bacterocera zonata under Laboratory Conditions. Egypt. Acad. J. Biol. Sci. (A: Entomol). 11, 3: 1-9.

Erwin, DG, Picton, BE, 1990: Guide to Inshore Marine Life. Immel Publishing. London.

Faulkner, DJ, 1992: Chemical defenses of marine molluscs. In: Ecological roles of marine natural products. New York: Cornwell University Press.

Fouda, MA, Hassan, MI, Hammad, KM, Hasaballah, AI, 2013: The Effects of Midgut Bacteria and Protease Inhibitors on the Reproductive Potential and Midgut Enzymes of Culex pipiens, mosquito Infected With Wuchereria Bancrofti filaria. J. Egypt. Soc. Parasitol. 43, 2: 537-545.

Geetha, DH, Rajeswari, M, Indhiramuthu, J, 2013: Chemical profiling of Elaeocarpus serratus L. by GC-MS. Asian Pac. J. Trop. Biomed. 3, 12: 985-7.

Wafa, G, Amadou, D, Larbi, KM, 2014: Larvicidal activity, phytochemical composition, and antioxidant properties of different parts of five populations of Ricinus communis. Ind. Crop. Prod. 56:43-51.

Gnanambal, KME, Chellaram, C, Jamila, P, 2005: Antibacterial activity of whole body extracts of Trochus radiatus (Mollusca: Gastropoda). In: Proc. Nat. Sem. Reef Ecosystem Remediation. SDMRI Res. Publica. 9:182-6

Gopalakrishnan, S, Vadivel, E, 2011: GC-MS Analysis of some bioactive constituents of Mussaenda frondosa Linn. Int. J. Pharm. Bio. Sci. 2, 1: 313-20.

Gurunathan, A, Senguttuvan, J, Paulsamy, S, 2016: Evaluation of mosquito repellent activity of isolated oleic acid, eicosyl ester from Thalictrum javanicum. Indian J. Pharm. Sci. 78, 1:103-10

Hasaballah, AI, El-Naggar, HA, 2017: Antimicrobial effects of some marine sponges, its biological and repellent activity against Culex pipiens (Diptera: Culicidae). Ann. Res. Rev. Biol. 12, 3: 1-14.

Hasaballah, AI, 2015: Toxicity of some plant extracts against vector of lymphatic filariasis, $\mathrm{Cu}$ - 
lex pipiens. J. Egypt. Soc. Parasitol. 45, 1:185-93. Ióca, LP, Nicacio, KJ, Berlinck, RG, 2018: Natural Products from Marine Invertebrates and Microorganisms in Brazil between 2004 and 2017: still the challenges, more rewards. J. Brazil. Chem. Soc. 29, 5:998-1031.

Jayashree, I, Geetha, DH, Rajeswari, M, 2015: GC-MS analysis of bioactive constituents of Glochidion ellipticum wt. Int. J. Pharm. Sci. Res. 6, 6:2546-50.

Jemimma, HL, Arumugasamy, K, Nantha, KR, Abdul-Kaffoor, H, 2017: GC-MS analysis of root and aerial parts ethanolic extract of Phyllanthus vasukii parthipan Et al, Sp. Nov. (Phyllanthaceae). Int. J. Ayurvedic. Herb 7, 4:2672-84. Karuso, P, 1987: Chemical ecology of the nudibranchs. Bioorg. Mar. Chem. 1:31-60.

Kegley, SE, Hill, BR, Orme, S, Choi, AH, 2010: Toxicity Information for Eugenol. PAN Pesticide Database. San Francisco, CA: Pesticide Action Network, North America.

Kumar, PS, Mishr, A, Malik, SS, 2012: Housefly Musca domestica L. control potential of Cymbopogon citratus Staff (Poales: Poaceae) essential oil and monoterpenes. Parasitol. Res. 112: 69-76.

Li, CH, Zhao, JM, Song, LS, 2009: A review of advances in research on marine molluscan antimicrobial peptides and their potential application in aquaculture. Molluscan Res. 29:17-26.

Lieske, E, Myers, RF, 2004: Coral Reef Guide Red Sea: The Definitive Guide to Over 1200 Species of Underwater Life. Harp. Collins, London.

Mather, JA, Mather, DL, 2004: Apparent movement in a visual display: the passing cloud of Octopus cyanea (Mollusca: Cephalopoda). J. Zool. Lond. 263, 1:89-94.

Miller, JH, Field, JJ, Kanakkanthara, A, Owen, JG, Singh, AJ, et al, 2018: Marine Invertebrate Natural Products that Target Microtubules. J. Nat. Prod. 81, 3:691-702.

Momtaz, ASK, 2016: Soft corals Sarcophyton glaucum as insecticide against Rice weevils Sitophilus oryzae (Coleoptera: Curculionidae). IOSR J. Environ. Sci. Toxicol. Food Technol. 10, 11: 10-4.

Nandita, C, Subrata, L, Goutam, C, 2008: Mosquito larvicidal and antimicrobial activity of protein of Solanum villosum leaves. BMC Compl. Alter. Med. 8:62.

Niyogi, P, Patinaik, S, Maharana, L, 2016: Quantitative identification of major and minor constituents of aerial parts of Mollugo pentaphylla Linn., using GC-MS. Asian J. Chem. 28, 10: 2335-8.
Ogino, T, Kurihara, C, Baba, Y, Kanematsu, K, 1994: Total Synthesis of Furoscrobiculin B. J. Chem. Soc. Chem. Commun. 19:79-80.

Panneerselvam, P, Sandhya, Kot, Vijayalakshmi, K, 2012: Dibenzoazepine, a pharmacologically active moiety. J. App. Chem. 1, 6:41-4.

Pavela, R, Maggi, F, Mbuntcha, H, Woguem, V, Fogang, HPD, et al, 2016: Traditional herbal remedies and dietary spices from Cameroon as novel sources of larvicides against filariasis mosquitoes? Parasitol. Res. 115, 12: 4617-26

Reegan, AD, Kinsalin, AV, Paulraj, MG, Ignacimuthu, S, 2013: Larvicidal, Ovicidal, and Repellent Activities of Marine Sponge Cliona celata (Grant) Extracts against Culex quinquefasciatus Say and Aedes aegypti L. (Diptera: Culicidae). ISRN Entomol. https://doi.org/10.1155/315389.

Singh, HB, Handique, AK, 1997: Antifungal activity of the essential oil of Hyptis suaveolens and its efficacy in biocontrol measures. J. Essen. Oil Res. 9, 6:683-7.

Song, MX, Zheng, CJ, Deng, XQ, Sun, LP, Wu, Y, et al, 2013: Synthesis and antibacterial evaluation of rhodanine-based 5-aryloxy pyrazoles against selected methicillin resistant and quinolone-resistant Staphylococcus aureus (MRSA and QRSA). Eur. J. Med. Chem. 60:376-85.

Sudhakar, S, Nazeer, R, 2015: Preparation of potent antioxidant peptide from edible part of shortclub cuttlefish against radical mediated lipid and DNA damage. LWT-Food Sci. Technol. 64: 593-601.

Uniyal, A, Tikar, SN, Mendki, MJ, Singh, R, Shukla, SV, et al, 2016: Behavioral Response of Aedes aegypti Mosquito towards Essential Oils Using Olfactometer. J. Arthropod Borne Dis. 10, 3: 370-80.

Vine, P, 1986: Red Sea Invertebrates. Immel Publishing, London.

WHO, 1996: Report of the WHO Informal Consultation on the Evaluation and Testing of Insecticides. CTD/WHOPES/IC/96.1.

Xiong, L, Li, J, Kong, F, 2004: Streptomyces sp. 173, an insecticidal microorganism from marine. Lett. Appl. Microbiol. 38:32-7

Zapata, A, Amemiya, CT, 2000: Phylogeny of lower vertebrates and their immunological structures. Curr. Trop. Microbiol. Immunol. 248:67107.

Zhao, Q, Li, Y, Xiong, L, Wang, Q, 2010: Design, synthesis and insecticidal activity of novel phenylpyrazoles containing a 2, 2, 2-trichloro-1alkoxyethyl moiety. J. Agric. Food Chem. 58: 4992-8. 